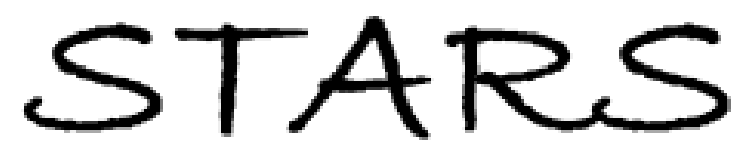

University of Central Florida

STARS

Faculty Bibliography 2000s

Faculty Bibliography

$1-1-2003$

\title{
Limiting aspect ratios of Sagnac interferometers
}

R. Glenn Sellar

University of Central Florida

Glenn D. Boreman

University of Central Florida

Find similar works at: https://stars.library.ucf.edu/facultybib2000

University of Central Florida Libraries http://library.ucf.edu

This Article is brought to you for free and open access by the Faculty Bibliography at STARS. It has been accepted for inclusion in Faculty Bibliography 2000s by an authorized administrator of STARS. For more information, please contactSTARS@ucf.edu.

\section{Recommended Citation}

Sellar, R. Glenn and Boreman, Glenn D., "Limiting aspect ratios of Sagnac interferometers" (2003). Faculty Bibliography 2000s. 4014.

https://stars.library.ucf.edu/facultybib2000/4014

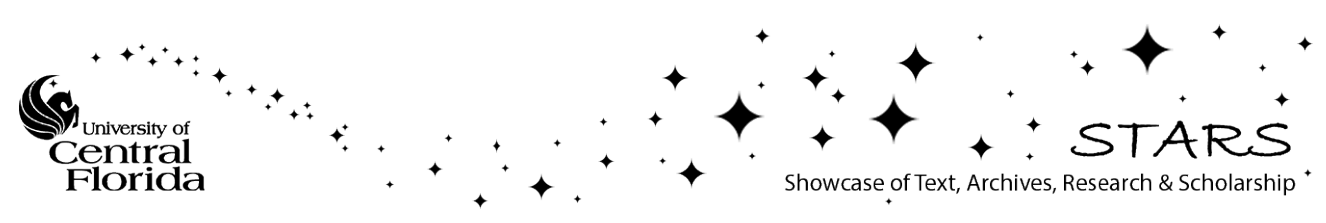




\section{Limiting aspect ratios of Sagnac interferometers}

\author{
R. Glenn Sellar, MEMBER SPIE \\ University of Central Florida \\ 12424 Research Parkway, Suite 400 \\ Orlando, Florida 32826-0650 \\ E-mail: gsellar@mail.ucf.edu
}

Glenn D. Boreman, FELLOW SPIE

University of Central Florida

CREOL, School of Optics

Orlando, Florida 32816-2700

\begin{abstract}
Any two-beam interferometer may be employed as a Fourier transform spectrometer. The two most commonly used for Fourier transform spectrometry are the Michelson interferometer and the Sagnac interferometer, the relative merits of which have been discussed in the literature. Typically, it is the interferometer that limits the acceptable range of angles for the input beam, and this maximum acceptance angle in turn limits the etendue, and hence limits the responsivity of the instrument when viewing an extended source. In designs where the interferometer is in a diverging or converging beam, the allowable range of input angles limits the focal ratio of the instrument, while in designs where the beam is collimated through the interferometer, this effect limits the fieldof-view of the instrument. In a Michelson, it is a loss of fringe contrast that limits the range of acceptance angles; a limitation that is discussed in many general texts on optics. A Sagnac, however, suffers no such loss of contrast as the range of acceptance angles is increased. The maximum acceptance angle for a Sagnac is instead limited by vignetting, caused by the geometry of the interferometer. The limitation for a Sagnac has an origin and behavior entirely different from that found for a Michelson, and has not been previously discussed in the literature. It is therefore important to understand this limitation when designing a Sagnac interferometer for Fourier transform spectrometry. This vignetting limitation may be quantified by an aspect ratio, which we define as the ratio of the separation of the entrance and exit apertures to the width of these apertures in the plane of the interferometer. To facilitate the design of Sagnac interferometers for Fourier transform spectrometry, we discuss the limitations on the aspect ratio and derive equations for the limiting aspect ratios for nine variations of the Sagnac interferometer. (C) 2003 Society of Photo-Optical Instrumentation Engineers. [DOI: 10.1117/1.1614810]
\end{abstract}

Subject terms: Sagnac interferometer; Fourier transform spectrometer; Fourier transform spectrometry; imaging spectrometer; imaging spectrometry.

Paper 030057 received Feb. 3, 2003; revised manuscript received May 15, 2003; accepted for publication May 16, 2003.

\section{Introduction}

While any two-beam interferometer may be used for Fourier transform spectrometry (FTS), the two most commonly used are the Michelson and Sagnac. The relative merits of the two have been discussed extensively in the literature in application to both nonimaging ${ }^{1-3}$ and imaging spectrometry. ${ }^{4-9}$ Briefly, the principal advantage of a FTS based on the Michelson is its ability to attain extremely high spectral resolution, while a FTS based on the Sagnac does not require a moving mirror and may therefore be relatively less expensive and more rugged.

Any interferometer of the two-beam, common-path type belongs to the family of Sagnac interferometers, ${ }^{10}$ though differences in the number and arrangement of the mirrors allow for an infinite number of variations within this family. Figure 1 shows a right-angle triangular Sagnac, comprised of the beamsplitter and two mirrors. One beam transits the interferometer in a clockwise direction, while the other beam transits the same path in a counterclockwise direction. In this arrangement, if the two mirrors were placed symmetrically with respect to the beamsplitter, then the two rays would exit the interferometer in the same direction and at the same position. If one mirror is offset from a symmetric position, however, then the two rays exit in the same direction, but at positions symmetrically offset from the optical axis. The path difference through the interferometer is a function of the angle of the ray entering the interferometer, but does not vary with the position of entry. The interferogram (fringe pattern) is localized at infinity, so a lens (referred to as the Fourier lens) is commonly used to image the fringes onto the detector array. The modulus of the Fourier transform of this interferogram gives the spectrum of the source. This arrangement provides the variation in path difference across the detector array with stationary mirrors, so there is no need for a scan mechanism.

Any beam passing through the interferometer must encounter the beamsplitter both on entering and exiting the interferometer. The beamsplitter therefore acts as both an entrance and an exit aperture, as shown in Fig. 2. Both entrance and exit apertures necessarily have the same dimensions, so if either aperture is used as the stop for the system, vignetting will occur at any angle greater than zero relative to the optic axis. As illustrated in Fig. 2(c), the angle at which the vignetting will reach $100 \%$ is determined by the aspect ratio of the interferometer, which we define as the ratio of the separation of the entrance and exit 


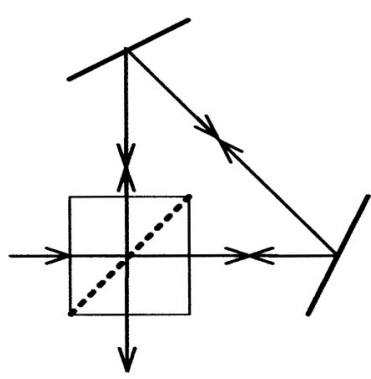

(a)

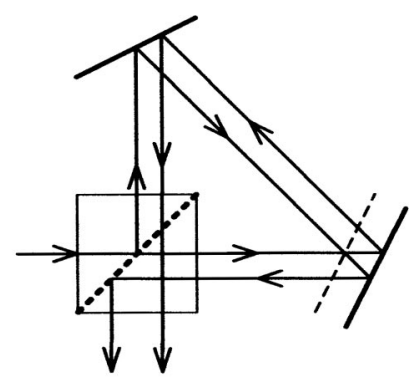

(b)
Fig. 1 Right-angle triangular Sagnac interferometer: (a) with mirrors in symmetrical positions, and (b) with lower mirror offset from the symmetrical position indicated by the dashed line.

apertures to the width of the apertures. In designs where the Sagnac is in a diverging beam, the aspect ratio limits the focal ratio; while in designs where the beam is collimated through the Sagnac, the aspect ratio limits the field of view (FOV). In the former case, the limitation on focal ratio limits the etendue, which in turn limits the responsivity of the instrument. Thus, for a designer to predict the responsivity, he or she must first determine this limitation on the focal ratio. In the latter case, it is vital for the designer to determine if the FOV required for the instrument exceeds the maximum FOV allowed for the interferometer. If this is the case, then additional measures must be taken to meet
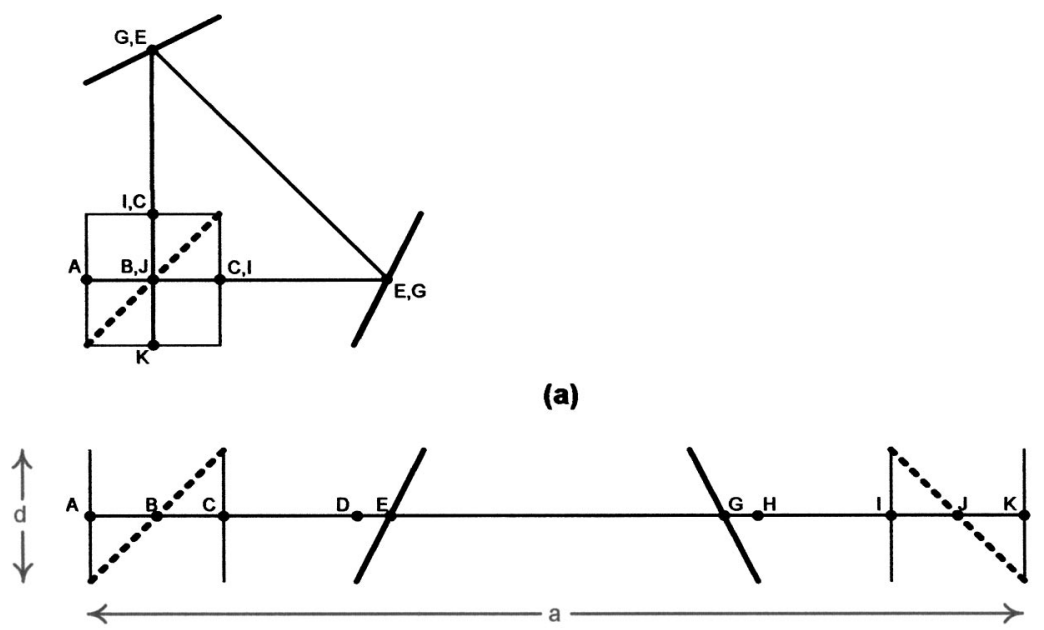

(b)

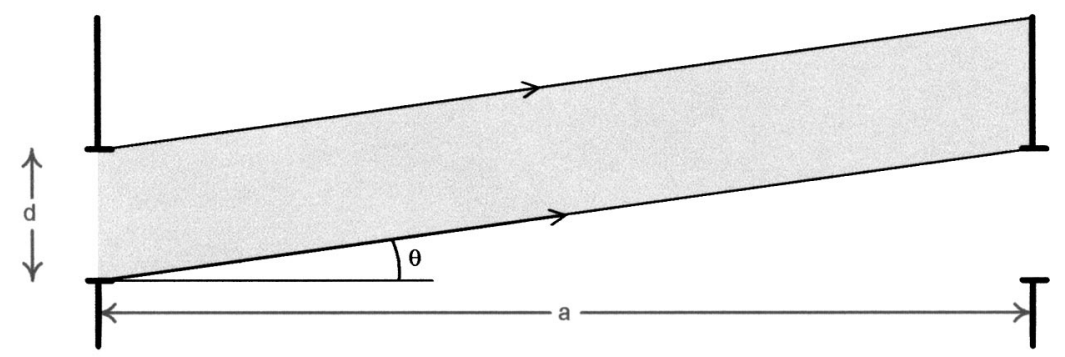

(c)

Fig. 2 (a) Layout of a right-angle triangular Sagnac interferometer showing the beamsplitter cube and two mirrors; (b) unfolded layout of the same interferometer; and (c) angle at which vignetting reaches $100 \%$. the requirement (for example, addition of a reversed afocal telescope in front of the interferometer to provide angular

In the case of a Michelson, this limitation results from a loss in fringe contrast, a discussion of which may be found in general optics texts. An understanding of the corresponding limitation for a Sagnac may be vital for a designer who is considering the use of a Sagnac for FTS, but this has not been previously discussed in the literature.

\section{Analysis}

\subsection{Limitations on the Aspect Ratio}

The aspect ratio $A / \#$ is defined as:

$A / \# \equiv \frac{a}{d}$,

where $d$ is the width of the entrance and exit apertures and $a$ is the unfolded distance between these apertures. The angle $\theta$ at which the vignetting will reach $100 \%$ is then given by:

$\theta=\arctan \left(\frac{1}{A / \#}\right)$. 

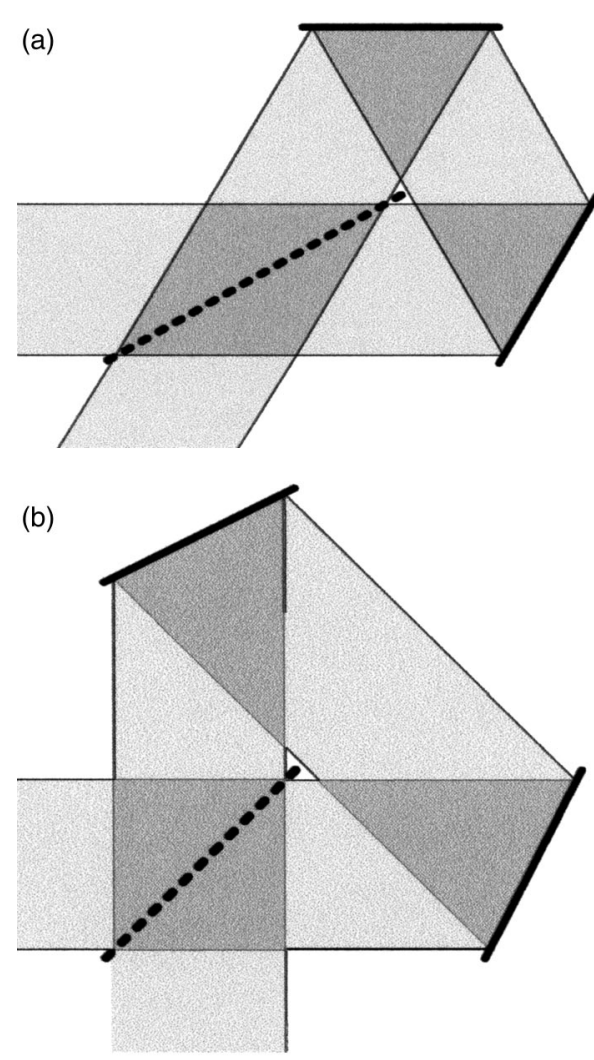

Fig. 3 (a) Aspect ratio for an equilateral-triangular Sagnac interferometer limited by vignetting of the beam between the two mirrors. (b) Aspect ratio for a right-angle triangular Sagnac interferometer limited by vignetting of the beam between the two mirrors.

The geometry of the interferometer, i.e., the number of mirrors and the arrangement of the mirrors and the beamsplitter, may impose limitations on the aspect ratio. For example, Fig. 3 shows plan views of Sagnacs formed as triangles, comprised of the beamsplitter and two mirrors. Note that in this case if we increase the width of the beamsplitter in the plane of the interferometer any further without increasing the separation of the elements, the beamsplitter will vignette the beam on its way from one mirror

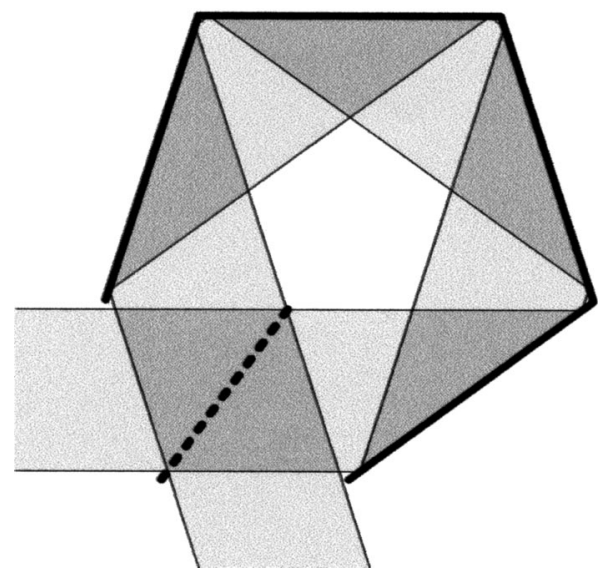

Fig. 4 Aspect ratio for a pentagonal Sagnac interferometer limited by mechanical interference between the mirrors.

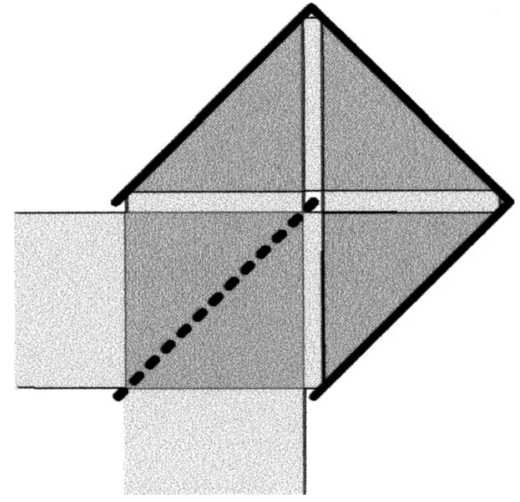

Fig. 5 Aspect ratio for a square Sagnac interferometer limited by vignetting of the beam between the two mirrors and by mechanical interference between the mirrors.

to the other mirror. Thus, the width of the aperture may only be increased if the separation of the elements is increased by the same factor. Such a modification will only change the scale of the system while maintaining the same aspect ratio. This limitation, however, does not apply to the height of the aperture, i.e., the dimension perpendicular to the plane of the interferometer. The aspect ratio is constrained only in the plane of the interferometer.

A regular pentagonal Sagnac, comprised of the beamsplitter and four mirrors, is shown in Fig. 4. Note that in this case edges of the mirrors are in contact with one another, and thus the width of the beam may not be increased any further. Once again, the width of the aperture may only be increased if the separation of the elements is increased by the same factor, and such a modification will merely change the scale while maintaining the same aspect ratio.

A square Sagnac is shown in Fig. 5. Note that in the case of a square Sagnac, both limitations-intrusion of the beamsplitter into the path between the mirrors and mechanical interference between the mirrors themselves-are reached simultaneously. Thus the aspect ratio of a regularpolygonal Sagnac with three or fewer mirrors will be limited by intrusion of the beamsplitter into the beam between the mirrors, and the aspect ratio with three or more mirrors will be limited by mechanical interference between the mirrors themselves.

\subsection{Limiting Aspect Ratio for a Right-Angle Triangular Sagnac}

The aspect ratio defined in Eq. (1) is a function of the distance between the entrance and exit apertures. For the right-angle triangular Sagnac shown in Fig. 2, the physical distance between the entrance and exit apertures as measured along the optic axis is:

$$
\begin{aligned}
a & \simeq \overline{A C}+\overline{C D}+\overline{D E}+\overline{E H}+\overline{H I}+\overline{I J}+\overline{J L}, \\
& =\overline{A C}+\overline{C D}+\overline{D E}+\sqrt{2}(\overline{B E})+\overline{D E}+\overline{C D}+\overline{A C}, \\
& =2 \overline{A C}+2 \overline{C D}+2 \overline{D E}+\sqrt{2}(\overline{B E}), \\
& =2 \overline{A C}+2 \overline{C D}+2 \overline{D E}+\sqrt{2}(\overline{B C}+\overline{C D}+\overline{D E}),
\end{aligned}
$$




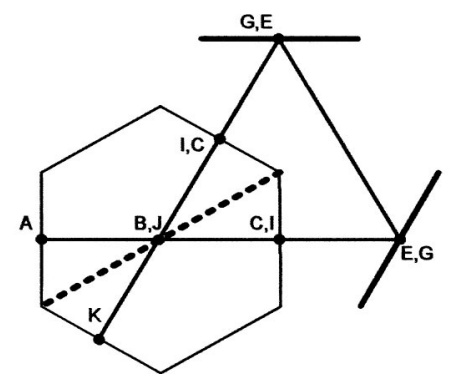

(a)

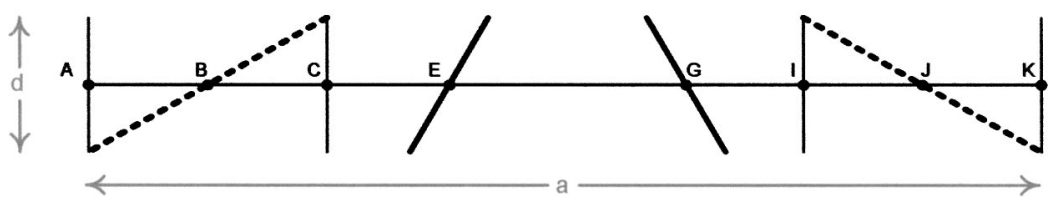

(b)

Fig. 6 (a) Layout of an equilateral-triangular Sagnac interferometer showing the beamsplitter prism and two mirrors; and (b) unfolded layout of the same interferometer.

$=2 d+2 d+d \tan \left(\frac{\pi}{8}\right)+\sqrt{2}\left[\frac{d}{2}+d+\frac{d}{2} \tan \left(\frac{\pi}{8}\right)\right]$,

$\simeq d(6.8)$

and the limiting aspect ratio is $A / \# \simeq 6.8$.

If any portion of the path between the entrance and exit apertures has a refractive index other than one, however, then the effective aspect ration for purposes of predicting the vignetting in Eq. (2) should use an effective separation. Using Snell's law and the small angle approximation, the effective separation through media with a refractive index $n$ should be divided by $n$. Thus if the beamsplitter cube illustrated in Fig. 2 has a refractive index $n$, then the effective separation $a$ becomes:

$$
\begin{aligned}
a & \simeq \frac{\overline{A C}}{n}+\overline{C D}+\overline{D E}+\overline{E H}+\overline{H I}+\overline{I J}+\frac{\overline{J L}}{n}, \\
& =\frac{\overline{A C}}{n}+\overline{C D}+\overline{D E}+\sqrt{2}(\overline{B E})+\overline{C D}+\overline{D E}+\frac{\overline{A C}}{n} \\
& =2\left(\frac{\overline{A C}}{n}\right)+2 \overline{C D}+2 \overline{D E}+\sqrt{2}(\overline{B E}), \\
& =2\left(\frac{\overline{A C}}{n}\right)+2 \overline{C D}+2 \overline{D E}+\sqrt{2}(\overline{B C}+\overline{C D}+\overline{D E}), \\
& =2\left(\frac{d}{n}\right)+2 d+d \tan \left(\frac{\pi}{8}\right)+\sqrt{2}\left[\frac{d}{2}+d+\frac{d}{2} \tan \left(\frac{\pi}{8}\right)\right] \\
& \simeq d\left(\frac{2}{n}+4.8\right),
\end{aligned}
$$

and the effective limiting aspect ratio is $A / \# \simeq 2 / n+4.8$.
In the case of a monolithic Sagnac, ${ }^{6}$ the entire interferometer is a single prism of refractive index $n$, and the effective separation is simply:

$a \simeq d\left(\frac{6.8}{n}\right)$

leading to an effective limiting aspect ratio of $A$ /\# $\simeq 6.8 / n$.

Finally, if a pellicle beamsplitter or a plate beamsplitter of negligible thickness is used, then the beamsplitter surface itself is the entrance and exit aperture and the separation is given by:

$$
\begin{aligned}
a & \simeq \overline{B C}+\overline{C D}+\overline{D E}+\overline{E H}+\overline{H I}+\overline{I J}+\overline{J K} \\
& =\overline{B C}+\overline{C D}+\overline{D E}+\sqrt{2}(\overline{B E})+\overline{D E}+\overline{C D}+\overline{B C} \\
& =2 \overline{B C}+2 \overline{C D}+2 \overline{D E}+\sqrt{2}(\overline{B E}) \\
& =2 \overline{B C}+2 \overline{C D}+2 \overline{D E}+\sqrt{2}(\overline{B C}+\overline{C D}+\overline{D E}), \\
& =d+2 d+d \tan \left(\frac{\pi}{8}\right)+\sqrt{2}\left[\frac{d}{2}+d+\frac{d}{2} \tan \left(\frac{\pi}{8}\right)\right] \\
& \simeq d(5.8)
\end{aligned}
$$

and the effective limiting aspect ratio is $A / \# \simeq 5.8$.

\subsection{Limiting Aspect Ratio for an Equilateral-Triangular Sagnac}

Following the notation shown in Fig. 6, for an equilateraltriangular Sagnac, the physical distance between the entrance and exit apertures as measured along the optic axis is: 


$$
\begin{aligned}
a & =\overline{A B}+\overline{B C}+\overline{C E}+\overline{E G}+\overline{G I}+\overline{I J}+\overline{J K}, \\
& =8 \overline{A B}, \\
& =8\left(\frac{\sqrt{3}}{2} d\right), \\
& \simeq d(6.9),
\end{aligned}
$$

and the limiting aspect ratio is $A / \# \simeq 6.9$.

If the beamsplitter is a hexagonal prism of refractive index $n$, then the effective separation $a$ becomes:

$$
\begin{aligned}
a & \simeq \frac{\overline{A B}}{n}+\frac{\overline{B C}}{n}+\overline{C E}+\overline{E G}+\overline{G I}+\frac{\overline{I J}}{n}+\frac{\overline{J K}}{n}, \\
& =4 \frac{\overline{A B}}{n}+4 \overline{A B}, \\
& =4\left(\frac{\frac{\sqrt{3}}{2} d}{n}\right)+4\left(\frac{\sqrt{3}}{2} d\right), \\
& \simeq d\left(\frac{3.46}{n}+3.46\right),
\end{aligned}
$$

and the effective limiting aspect ratio is $A / \# \simeq 3.46 / n$ +3.46 .

If the equilateral-triangular Sagnac is monolithic, then

$a \simeq d\left(\frac{6.9}{n}\right)$,

leading to an effective limiting aspect ratio of $A$ /\# $\simeq 6.9 / n$

Finally, if a pellicle beamsplitter or a plate beamsplitter of negligible thickness is used in an equilateral-triangular Sagnac, then

$$
\begin{aligned}
a & \simeq \overline{B C}+\overline{C E}+\overline{E G}+\overline{G I}+\overline{I J}, \\
& =6 \overline{A B}, \\
& =6\left(\frac{\sqrt{3}}{2} d\right), \\
& \simeq d(5.2),
\end{aligned}
$$

and the effective limiting aspect ratio is $A / \# \simeq 5.2$.

\subsection{Limiting Aspect Ratio for a Square Sagnac}

Following the notation shown in Fig. 7, for a square Sagnac the physical distance between the entrance and exit apertures as measured along the optic axis is:

$$
\begin{aligned}
a & =\overline{A B}+\overline{B C}+\overline{C E}+\overline{E F}+\overline{F G}+\overline{G I}+\overline{I J}+\overline{J K}, \\
& =10 \overline{A B},
\end{aligned}
$$

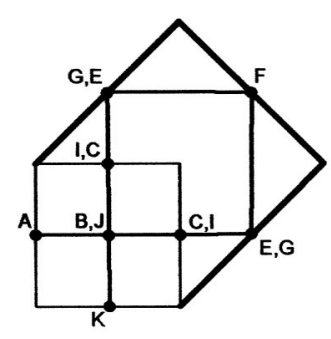

(a)

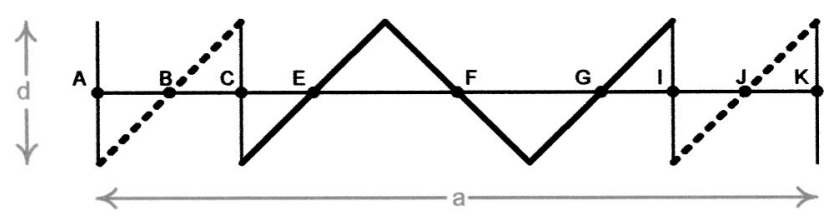

(b)

Fig. 7 (a) Layout of a square Sagnac interferometer showing the beamsplitter cube and three mirrors; and (b) unfolded layout of the same interferometer.

$$
\begin{aligned}
& =10\left(\frac{d}{2}\right), \\
& =d(5),
\end{aligned}
$$

and the limiting aspect ratio is $A / \#=5$.

If the beamsplitter is a cube of refractive index $n$, then the effective separation $a$ becomes:

$$
\begin{aligned}
a & \simeq \frac{\overline{A B}}{n}+\frac{\overline{B C}}{n}+\overline{C E}+\overline{E F}+\overline{F G}+\overline{G I}+\frac{\overline{I J}}{n}+\frac{\overline{J K}}{n}, \\
& =5 \frac{\overline{A B}}{n}+5 \overline{A B} \\
& =5\left(\frac{d / 2}{n}\right)+5(d / 2), \\
& =d\left(\frac{2.5}{n}+2.5\right),
\end{aligned}
$$

and the effective limiting aspect ratio is $A / \#=2.5 / n+2.5$.

If the square Sagnac is monolithic, then

$a \simeq d\left(\frac{5}{n}\right)$,

leading to an effective limiting aspect ratio of $A / \# \simeq 5 / n$.

Finally, if a pellicle beamsplitter or a plate beamsplitter of negligible thickness is used in a square Sagnac, then

$$
\begin{aligned}
a & =\overline{B C}+\overline{C E}+\overline{E F}+\overline{F G}+\overline{G I}+\overline{I J}, \\
& =8 \overline{A B}, \\
& =8\left(\frac{d}{2}\right), \\
& =d(4),
\end{aligned}
$$


Table 1 Limiting aspect ratios for nine variations of the Sagnac interferometer.

\begin{tabular}{lccc}
\hline \hline Geometry & $\begin{array}{c}\text { Equilateral } \\
\text { triangular }\end{array}$ & $\begin{array}{c}\text { Right-angle } \\
\text { triangular }\end{array}$ & Square \\
\hline $\begin{array}{l}\text { Beamsplitter type } \\
\text { Cube or prism with } \\
\text { refractive index } n\end{array}$ & $\frac{3.46}{n}+3.46$ & $\frac{2}{n}+4.8$ & $\frac{2.5}{n}+2.5$ \\
$\begin{array}{l}\text { Monolithic with } \\
\text { refractive index } n\end{array}$ & $\frac{6.9}{n}$ & $\frac{6.8}{n}$ & $\frac{5}{n}$ \\
Pellicle or thin plate & 5.2 & 5.8 & 4 \\
\hline \hline
\end{tabular}

and the effective limiting aspect ratio is $A / \#=4$.

\section{Conclusions}

The limiting aspect ratios for nine configurations of the Sagnac interferometer are listed in Table 1. In designs where the Sagnac is in a converging beam, the aspect ratio limits the focal ratio; in designs where the beam is collimated through the Sagnac, the aspect ratio limits the field of view. The aspect ratio in the plane of the interferometer is limited by the geometry of the interferometer. For example, for a right-angle triangular cube with $n=1.5$, the $A / \#=6.1$ and vignetting reaches $100 \%$ at $9.3 \mathrm{deg}$. Due to the wide range of applications for imaging spectrometers, the required FOV may range from less than one degree to 10 's of degrees, so the restriction on the aspect ratio may or may not be significant for a particular application. It should also be noted once again that, as the aspect ratio in the direction perpendicular to the plane of the interferometer is not limited, there is no restriction on the focal ratio or the field of view in the direction perpendicular to the plane of the interferometer. A difference in the extent of the FOV in these two directions, however, will produce a rectangular (rather than square) image on the array. If the difference in the FOVs is large, this may lead to inefficient use of the detector array, since most available arrays have a 1:1 or 3:4 ratio of height to width. If a designer, using the aspect ratios presented in Table 1, determines that the vignetting caused by these limitations on the aspect ratio would make it impossible to meet the FOV requirement for the instrument, one possible remedy is to add a reversed afocal telescope in front of the interferometer to provide angular demagnication. ${ }^{9}$ One application of the analyses presented here is to enable a designer to determine whether such a remedy is necessary.

\section{Acknowledgment}

This research was funded in part by NASA under grant number NAG5-10730.

\section{References}

1. G. W. Stroke and A. T. Funkhouser, Phys. Lett. 16, 272 (1965).

2. K. Yoshihara and A. Kitade, "Holographic spectra using a triangle path interferometer," Jpn. J. Phys. 6, 116 (1967).

3. T. Okamoto, S. Kawata, and S. Minami, "Fourier transform spectrometer with a self-scanning photodiode array," Appl. Opt. 23, 269 (1984).

4. P. G. Lucey, K. A. Horton, T. J. Williams, K. Hinck, C. Budney, J. B. Rafert, and T. B. Rusk, "SMIFTS: A cryogenically cooled, spatially modulated imaging infrared interferometer spectrometer," Proc. SPIE 1937, 130-141 (1993)

5. R. G. Sellar and J. B. Rafert, "The effects of aberrations on spatially modulated Fourier transform spectrometers," Opt. Eng. 33(9), 30873092 (1994).

6. J. B. Rafert and R. G. Sellar, "Monolithic Fourier transform imaging spectrometer," Appl. Opt. 34, 7228-7230 (1995)

7. L. J. Otten, J. B. Rafert, A. Meigs, B. Jones, R. Prinzing, R. Fronterhouse, R. G. Sellar, and P. Hodge, "The engineering model for the MightySat II. 1 hyperspectral imager," Proc. SPIE 3221, 412-420 (1997).

8. W. J. Slough, J. B. Rafert, C. A. Rohde, and C. L. Hart, "THRIFTI: Tomographic hyperspectral remote imaging Fourier transform interferometer," Proc. SPIE 3393, 207-216 (1998).

9. R. G. Sellar, "The optical engineering of imaging spectrometers based on the Sagnac interferometer," Doctoral Dissertation, University of Central Florida (2003).

10. M. G. Sagnac, "Effect tourbillonaire optique la circulation de l'éther lumineux dans un interférographe tournant," J. Physique Thâeorique et Appliquâee 5(4), 177-195 (1914).

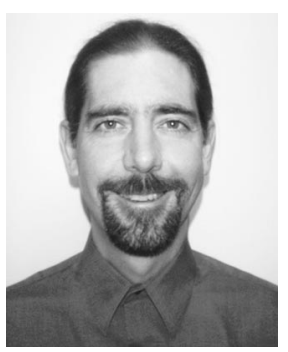

R. Glenn Sellar has 16 years of experience in the design, integration, and testing of imaging spectrometers for groundbased, airborne, and spaceborne applications. He worked as an optical engineer on the Wind Imaging Interferometer (WINDII) for the Upper Atmosphere Research Satellite (UARS) and designed the optical system for the Fourier Transform HyperSpectral Imager (FTHSI) on MightySat II. He received a BEng degree in engineering physics from McMaster University in 1986, an MS degree in space sciences from the Florida Institute of Technology in 1993, and a PhD degree in optics from the University of Central Florida in 2003. He is a member of SPIE. He is currently developing imaging spectrometers for planetary science and earth science applications at the University of Central Florida.

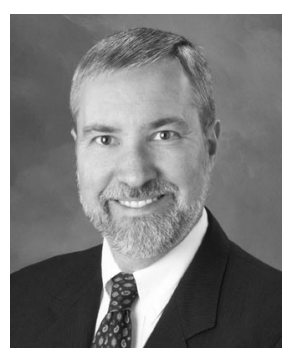

Glenn D. Boreman received a BS in optics from Rochester and a PhD in Optics from Arizona. Since 1984, he has been on the faculty of the University of Central Florida, where he is a Professor of Optics and Electrical Engineering. He currently serves as the Editor-in-Chief of Applied Optics, and is a past member of the SPIE Board of Directors. He is coauthor of Infrared Detectors and Systems (Wiley), author of Basic Electro-Optics for Electrical Engineers, and Modulation Transfer Function in Optical and Electro-Optical Systems, and editor of Infrared Technology 1988-1998, all from SPIE Press. He is a fellow of OSA and SPIE. Along with his students, he received the 1995 Kingslake Medal from SPIE. 